\title{
High Order Fefferman-Phong Type Inequalities in Carnot Groups and Regularity for Degenerate Elliptic Operators plus a Potential
}

\author{
Pengcheng Niu and Kelei Zhang \\ Department of Applied Mathematics, Northwestern Polytechnical University, Xian, Shaanxi 710129, China \\ Correspondence should be addressed to Pengcheng Niu; pengchengniu@nwpu.edu.cn
}

Received 4 June 2014; Accepted 13 October 2014; Published 10 November 2014

Academic Editor: Sung G. Kim

Copyright (C) 2014 P. Niu and K. Zhang. This is an open access article distributed under the Creative Commons Attribution License, which permits unrestricted use, distribution, and reproduction in any medium, provided the original work is properly cited.

Let $\left\{X_{1}, X_{2}, \ldots, X_{m}\right\}$ be the basis of space of horizontal vector fields in a Carnot group $\mathbb{G}=\left(\mathbb{R}^{n} ; \circ\right)(m<n)$. We prove high order Fefferman-Phong type inequalities in $\mathbb{G}$. As applications, we derive a priori $L^{p}(\mathbb{G})$ estimates for the nondivergence degenerate elliptic operators $L=-\sum_{i, j=1}^{m} a_{i j}(x) X_{i} X_{j}+V(x)$ with $V M O$ coefficients and a potential $V$ belonging to an appropriate Stummel type class introduced in this paper. Some of our results are also new even for the usual Euclidean space.

\section{Introduction and the Main Results}

The classical $L^{p}$ estimates for nondivergence elliptic operators with potentials of the form

$$
\mathfrak{L} u \equiv-\sum_{i, j=1}^{n} a_{i j}(x) u_{x_{i} x_{j}}+V u, \quad x \in \mathbb{R}^{n}
$$

have been extensively investigated and many results have been proved; see [1-5] and so forth. In particular, when $\left(a_{i j}\right)_{n \times n}=I$, the identity matrix, and $V$ belongs to the reverse Hölder class $B_{q}(n / 2 \leq q<\infty)$, Shen [2] established $L^{p}(1<$ $p \leq q)$ boundedness for the Schrödinger operator $-\Delta+V$ and showed that the range of $p$ is optimal. It is noted that $V \in B_{q}(q>1)$ means that $V \in L_{\text {loc }}^{q}\left(\mathbb{R}^{n}\right), V \geq 0$, and there exists a positive constant $c$ such that the reverse Hölder inequality

$$
\left(|B|^{-1} \int_{B} V^{q}(x) d x\right)^{1 / q} \leq c\left(|B|^{-1} \int_{B} V(x) d x\right)
$$

holds for every ball $B$ in $\mathbb{R}^{n}$. More recently, when $V \in$ $B_{q}(n / 2 \leq q<\infty)$, a priori $L^{p}\left(\mathbb{R}^{n}\right)(1<p \leq q)$ estimate for $\mathbb{L}$ in (1) with $V M O$ coefficients has been deduced by
Bramanti et al. [1] by using the representation formula for $V u$ in terms of $\mathfrak{Q} u$, which generalized the result in [2]. The aim of this paper is to establish high order Fefferman-Phong type inequalities in Carnot groups and prove $L^{p}$ regularity of degenerate elliptic operators plus a potential.

Let $X_{1}, X_{2}, \ldots, X_{m}$ be horizontal vector fields in a Carnot group $\mathbb{G}=\left(\mathbb{R}^{n} ; \circ\right),(m<n)$ (see Section 2.1). In this paper we consider the nondivergence degenerate elliptic operator of the kind

$$
L u \equiv A u+V u \equiv-\sum_{i, j=1}^{m} a_{i j}(x) X_{i} X_{j} u+V u,
$$

where the leading coefficient $a_{i j}(x)$ satisfies $a_{i j}(x)=a_{j i}(x) \epsilon$ $L^{\infty}(\mathbb{G})$ for $i, j=1, \ldots, m$, and there exists a constant $\mu>0$ such that, for any $x \in \mathbb{G}$ and $\xi \in \mathbb{R}^{m}$,

$$
\mu|\xi|^{2} \leq \sum_{i, j=1}^{m} a_{i j}(x) \xi_{i} \xi_{j} \leq \mu^{-1}|\xi|^{2}
$$

furthermore, we assume

$$
a_{i j}(x) \in \operatorname{VMO}(\mathbb{G}),
$$


which shows that, for $i, j=1, \ldots, m$,

$$
\begin{array}{r}
\eta_{i j}=\sup _{\rho \leq r} \sup _{x \in \mathbb{G}}\left(\left|B_{\rho}(x)\right|^{-1} \int_{B_{\rho}(x)}\left|a_{i j}(y)-a_{i j}^{B}\right| d y\right) \\
r \longrightarrow 0, \\
r \longrightarrow 0^{+} .
\end{array}
$$

Here $a_{i j}^{B}=\left|B_{\rho}(x)\right|^{-1} \int_{B_{\rho}(x)} a_{i j}(y) d y \cdot B_{\rho}(x)$ denotes a metric ball of radius $r$ and center $x$ associated with the CarnotCarathéodory distance $d$ (see Section 2) by

$$
B_{\rho}(x)=B(x, \rho)=\{y \in \mathbb{G}: d(x, y)<\rho\} .
$$

As to the potential $V$, inspired by Di Fazio and Zamboni [6, Definition 2.4], we introduce the following Stummel type class $S_{p}$.

Definition 1 (Stummel type class). Let $V: \mathbb{G} \rightarrow \mathbb{R}, 1<p<$ $\infty, r>0$. One says that $V \in S_{p}(\mathbb{G})$, if for every $r>0$,

$$
\begin{aligned}
& \varphi_{V}(r):=\sup _{x \in \mathbb{G}}\left(\int_{d(x, y)<r} \frac{d(x, y)^{2}}{|B(x, d(x, y))|}\right. \\
& \quad \times\left(\int_{d(z, x)<r}|V(z)|\right. \\
&\left.\left.\quad \times \frac{d(z, y)^{2}}{|B(z, d(z, y))|} d z\right)^{1 /(p-1)} d y\right)^{p-1}
\end{aligned}
$$

is finite and

$$
\lim _{r \rightarrow 0} \varphi_{V}(r)=0
$$

where $d(\cdot, \cdot)$ is the Carnot-Carathéodory distance; see Section 2.

Sometimes we will call $\varphi_{V}(r)$ the Stummel modulus of $V$.

Remark 2. We note that $L^{\infty}(\mathbb{G}) \subset S_{p}(\mathbb{G})(1<p<\infty)$ and $S_{p}(\mathbb{G})$ is the special case of the function class in [7, page 56] with $p=2$ and $\mathbb{G}=\mathbb{R}^{n}(n \geq 5)$. Also, note that the function $V(x)=d(0, x)^{-2}$ on $\mathbb{G}$ belongs to the classes $S_{p}(\mathbb{G})$ for $p>2$, where $d$ is the Carnot-Carathéodory distance (see Section 2).

Nondivergence degenerate elliptic operators similar to (3) including the form $-\sum_{i=1}^{m} X_{i}^{2}+V$ have been studied by some authors; see [8-11] and so forth. The local $L^{p}$ estimate for operator (3) with the vanishing potential $V=0$ on the homogeneous group has been verified by Bramanti and Brandolini [12]. For the study of related operators, we refer to $[13,14]$ and references therein. We will prove regularity for the operator $L$ in (3) on $\mathbb{G}$ if $a_{i j}$ satisfy (4)-(5) and $V^{p} \in$ $S_{p}(\mathbb{G})$; see Theorem 3 below. Our methods are different from the Euclidean case by Bramanti et al. [1], where estimates of integral operators and their commutators were used as a main tool.
Since Fefferman [15] proved the well-known imbedding inequality

$$
\int_{\mathbb{R}^{n}}|V||u|^{2} d x \leq c \int_{\mathbb{R}^{n}}|\nabla u|^{2} d x, \quad u \in C_{0}^{\infty}\left(\mathbb{R}^{n}\right),
$$

with $V$ belonging to the classical Morrey class $L^{r, n-2 r}, 1<r \leq$ $n / 2$, it has been extended to many more general settings and applied to infer regularity for partial differential operators; see $[6,16-19]$ and so forth. One of the main jobs of this paper is to establish a high order Fefferman-Phong type inequality in Carnot groups (see Theorem 4), which is motivated by Di Fazio and Zamboni [6, Theorem 3.1]. So far as we know, there is not any result in literature on high order FeffermanPhong inequalities. Using this inequality and proving several estimates with the potential, a priori $L^{P}(\mathbb{G})$ estimate for $L$ is obtained.

We mention that the homogeneous dimension $Q$ of $\mathbb{G}$, the horizontal gradient $X u$, the second order horizontal gradient $X^{2} u$, the horizontal Sobolev spaces $H W^{2, p}(\mathbb{G})$ and $H W_{V}^{2, p}(\mathbb{G})$, the polynomial $P_{B}(x)$, and the reverse Hölder class $B_{q}$ in our setting will be described in Section 2. Now we are in a position to state main results.

Theorem 3. Under the assumptions (4)-(5), if $V^{p} \in S_{p}(\mathbb{G}), 1<$ $p<\infty$, then there exists a positive constant $c=c(p, \mu, \eta, V, \mathbb{G})$ such that, for any $u \in C_{0}^{\infty}(\mathbb{G})$, it follows that

$$
\begin{aligned}
& \|u\|_{H W^{2, p}(\mathbb{G})}+\|V u\|_{L^{p}(\mathbb{G})} \\
& \quad \leq c\left(\|L u\|_{L^{p}(\mathbb{G})}+\|u\|_{L^{p}(\mathbb{G})}\right),
\end{aligned}
$$

where $\eta$ in $c$ depends only on the VMO moduli $\eta_{i j}$ of the coefficients $a_{i j}$. Furthermore, (11) holds for $u \in H W_{V}^{2, p}(\mathbb{G})$.

It is noted that the $L^{p}$ estimates of the operators similar to (3) with discontinuous leading coefficients and bounded lower terms were obtained by Bramanti and Brandolini [12, 20]. Here the potential $V$ in Theorem 3 may be unbounded on $\mathbb{G}$.

The key for the proof of Theorem 3 is the following high order Fefferman-Phong type inequality.

Theorem 4. Let $B=B_{r_{B}}\left(x_{0}\right)$ be any metric ball in $\mathbb{G}$. If $V \in$ $S_{p}(\mathbb{G})(1<p<\infty)$, then there exists a first order polynomial $P_{B}(x)$ such that, for any $u \in C^{\infty}(B)$, one has

$$
\int_{B}\left|u-P_{B}(x)\right|^{p}|V| d x \leq c \varphi_{V}\left(2 r_{B}\right) \int_{B}\left|X^{2} u\right|^{p} d x
$$

where the positive constant $c$ is independent of $u$ and $B$. Moreover, for any $u \in C_{0}^{\infty}(B)$, one has

$$
\int_{B}|u|^{p}|V| d x \leq c \varphi_{V}\left(2 r_{B}\right) \int_{B}\left|X^{2} u\right|^{p} d x,
$$

where $c>0$ is independent of $u$ and $B$.

The above $X^{2} u$ is a set of $X_{i} X_{j} u$ for all $i, j=1, \ldots, m$. We will define $X^{2} u$ precisely in Section 2. 
Remark 5. The main difference between Theorem 4 and [6, Theorem 3.1] is clear; that is, the right-hand side term $\|X u\|_{L^{p}(B)}$ in [6] is replaced by $\left\|X^{2} u\right\|_{L^{p}(B)}$ here. Of course, the class involving $V$ is not the same.

We observe an important relation between the Stummel class here and the reverse Hölder class: if $V \in B_{q} \cap L^{1}(\mathbb{G})$, $q>Q / 2$, then $V^{p} \in S_{p}(\mathbb{G}), 1<p \leq 2 q / Q$. From it and Theorem 3 , the following result follows.

Theorem 6. Under the same assumptions on $a_{i j}$ as in Theorem 3, if $V \in B_{q} \cap L^{1}(\mathbb{G}), q>Q / 2$, then for $1<p \leq 2 q / Q$ and $u \in C_{0}^{\infty}(B)$, the estimate (11) holds.

Remark 7. When $q=Q / 2$ and $V \in B_{\mathrm{Q} / 2} \bigcap L^{1}(\mathbb{G})$, by the important property of the $B_{q}$ class (see [21]), there exists $\varepsilon>0$ such that $V \in B_{\mathrm{Q} / 2+\varepsilon}$. Therefore, estimate (11) holds for $1<p \leq 1+2 \varepsilon / Q$ and $u \in C_{0}^{\infty}(B)$.

The paper is organized as follows. In Section 2 we recall some basic facts about Carnot groups and function spaces. In Section 3 we first give the proof of Theorem 4. Then combining with the known result in [12, Theorem 2] and proving an estimate with the potential $V$, we finish the proof of Theorem 3. The proof of Theorem 6 is given in Section 4. In Section 5, we restate Theorems 3 and 4 for the Euclidean case and elliptic operators without proofs.

Dependence of Constants. Throughout this paper, the letter $c$ denotes a positive constant which may vary from line to line.

\section{Preliminaries}

2.1. Background on Carnot Groups. We collect some facts about Carnot groups that will be needed in the sequel and refer the readers to [22-25] for further details.

Definition 8 (Carnot group). A Carnot group $\mathbb{G}=\left(\mathbb{R}^{n} ; \circ\right)$ is a simply connected nilpotent Lie group such that its Lie algebra $\widetilde{g}$ admits a stratification

$$
\widetilde{g}=V_{1} \oplus V_{2} \oplus \cdots \oplus V_{r}=\oplus_{j=1}^{r} V_{j},
$$

where $\left[V_{1}, V_{j}\right]=V_{j+1}, j=1, \ldots, r-1$, and $\left[V_{1}, V_{r}\right]=\{0\}$. Here $r$ is called the step of $\mathbb{G}$.

For $k=1, \ldots, r$, let $X_{1, k}, \ldots, X_{m_{k}, k}$ be a basis of $V_{k}$ consisting of commutators of length $k$, where $m_{k}$ is the dimension of $V_{k}$. The horizontal vector fields are ones in the first layer $V_{1}$ and for convenience, we set $m_{1}=m$ and denote $X_{i, 1}=$ $X_{i}, i=1, \ldots, m$. Clearly, vector fields $X_{1}, \ldots, X_{m}$ satisfy Hormander's condition [26].

Let $\left\{\delta_{\lambda}\right\}_{\lambda>0}$ be a family of nonisotropic dilations on $\mathbb{G}$ defined by

$$
\delta_{\lambda}: \mathbb{G} \longrightarrow \mathbb{G}, \quad \delta_{\lambda}(\xi)=\left(\lambda \xi_{1}, \lambda^{2} \xi_{2}, \ldots, \lambda^{r} \xi_{r}\right)
$$

for any $\lambda>0$ and $\xi=\left(\xi_{1}, \ldots, \xi_{r}\right)=\left(x_{1,1}, \ldots, x_{m_{1}, 1}, \ldots\right.$, $\left.x_{1, r}, \ldots, x_{m_{r}, r}\right) \in \mathbb{G}$. The integer $Q=\sum_{k=1}^{r} k m_{k}$ is said to be the homogeneous dimension of $\mathbb{G}$. In general, we assume $Q \geq 4$. We call that a vector field $X_{i, k} \in \widetilde{g}$ is left invariant if for any smooth function $f$ one has

$$
X_{i, k}^{x}(f(y \circ x))=\left(X_{i, k} f\right)(y \circ x), \quad \text { for any } x \in \mathbb{G},
$$

and $X_{i, k}$ is sth homogeneous if for any smooth function $f$, it follows that

$$
X_{i, k}\left(f\left(\delta_{\lambda}(x)\right)\right)=\lambda^{s}\left(X_{i, k} f\right)\left(\delta_{\lambda}(x)\right), \quad \text { for any } x \in \mathbb{G} .
$$

As in [23], the homogeneous norm of $\xi \in \mathbb{G}$ is defined by

$$
d_{\mathbb{G}}(\xi)=\left[\sum_{k=1}^{r}\left(\sum_{i=1}^{m_{k}}\left|\xi_{i, k}\right|^{2}\right)^{r ! / k}\right]^{1 / 2 r !} .
$$

It is natural to define the pseudo distance by the homogeneous norm

$$
d_{\mathbb{G}}(\xi, \eta)=d_{\mathbb{G}}\left(\eta^{-1} \circ \xi\right), \quad \text { for any } \xi, \eta \in \mathbb{G},
$$

where $\eta^{-1}$ is the inverse of $\eta$. A polynomial on $\mathbb{G}[24]$ is a function which can be expressed in exponential coordinates (see, e.g., [22, Section 2.1.9] and [27]) as

$$
P(x)=\sum_{I} a_{I} x^{I}, \quad a_{I} \in \mathbb{R},
$$

where $I=\left(i_{j, k}\right)_{j=1, \ldots, m_{k}}^{k=1, \ldots, r}$ are multi-indices and

$$
x^{I}=\prod_{k=1, \ldots, r ; j=1, \ldots, m_{k}} x_{j, k}^{i_{j}, k} .
$$

The homogeneous degree of monomial $x^{I}$ is the sum $d(I)=$ $\sum_{k=1}^{r} \sum_{j=1}^{m_{k}} k i_{j, k}$ and the homogeneous degree of $P(x)$ is $\max \left\{d(I) \mid a_{I} \neq 0\right\}$.

From [28], the left invariant vector fields $X_{1}, \ldots, X_{m}$ can induce the corresponding Carnot-Carathéodory distance $d$ : for any $\delta>0$, let $A(\delta)$ be the set of absolutely continuous curves $\gamma:[0,1] \rightarrow \mathbb{G}$ such that for a.e. $t \in[0,1]$,

$$
\begin{aligned}
& \gamma^{\prime}(t)=\sum_{i=1}^{m} a_{i}(t) X_{i}(\gamma(t)) \\
& \text { with } \sum_{i=1}^{m}\left|a_{i}(t)\right| \leq \delta .
\end{aligned}
$$

By [29], it is known that for $\delta$ large enough the set $A(\delta)$ is nonempty. We define the Carnot-Carathéodory distance by

$$
d(\xi, \eta)=\inf \{\delta>0 \mid \exists \delta \in A(\delta) \text { with } \gamma(0)=\xi, \gamma(1)=\eta\} \text {. }
$$

It is well known that the distance $d$ is equivalent to the pseudo distance $d_{\mathbb{G}}$ (see [28]). In this paper, we will mainly use the Carnot-Carathéodory distance $d$ to study regularity of (3). 
Associated with the distance, we define the metric ball of center $\xi$ and radius $r$ in $\mathbb{G}$ by

$$
B_{r}(\xi)=\{\eta \in \mathbb{G} \mid d(\xi, \eta)<r\}
$$

The Lebesgue measure in $\mathbb{R}^{n}$ is the Haar measure on $\mathbb{G}$ ([25, page 619]). Due to (15), one has

$$
\left|B_{r}(\xi)\right|=C_{Q^{2}}{ }^{Q}
$$

where $\left|B_{r}(\xi)\right|$ is the measure of $B_{r}(\xi)$ and $C_{\mathrm{Q}}$ is a positive constant.

2.2. Function Spaces. Denote $X=\left(X_{1}, \ldots, X_{m}\right), X u=$ $\left(X_{1} u, \ldots, X_{m} u\right), X^{2} u=\left\{X_{i} X_{j} u\right\}_{i, j=1}^{m},|X u|=\sum_{i=1}^{m}\left|X_{i} u\right|$, and $\left|X^{2} u\right|=\sum_{i, j=1}^{m}\left|X_{i} X_{j} u\right|$.

Definition 9 (Horizontal Sobolev space). For any $p \geq 1$ and a domain $\Omega \subseteq \mathbb{G}$, one defines the Horizontal Sobolev spaces by

$$
H W^{2, p}(\Omega)=\left\{u \in L^{p}(\Omega) \mid\|u\|_{H W^{2, p}(\Omega)}<+\infty\right\}
$$

with the norm

$$
\|u\|_{H W^{2, p}(\Omega)}=\|u\|_{L^{p}(\Omega)}+\|X u\|_{L^{p}(\Omega)}+\left\|X^{2} u\right\|_{L^{p}(\Omega)},
$$

where $\|X u\|_{L^{p}(\Omega)}=\sum_{i=1}^{m}\left\|X_{i} u\right\|_{L^{p}(\Omega)},\left\|X^{2} u\right\|_{L^{p}(\Omega)}=$ $\sum_{i, j=1}^{m}\left\|X_{i} X_{j} u\right\|_{L^{p}(\Omega)}$.

Analogously to [1], the space $H W_{V}^{2, p}(\Omega)$ is the closure of $C_{0}^{\infty}(\Omega)$ in the norm

$$
\|u\|_{H W_{V}^{2, p}(\Omega)}=\|u\|_{H W^{2, p}(\Omega)}+\|V u\|_{L^{p}(\Omega)}
$$

Definition 10 (Reverse Hölder class). (1) A nonnegative locally $L^{q}$ integrable function $V(x)$ on $\mathbb{G}$ is said to belong to the reverse Hölder class $B_{q}(1<q<\infty)$, if there exists a positive constant $c$ such that

$$
\left(|B|^{-1} \int_{B} V^{q}(x) d x\right)^{1 / q} \leq c\left(|B|^{-1} \int_{B} V(x) d x\right),
$$

for any metric ball $B$ in $\mathbb{G}$.

(2) Let $V(x)>0$ a.e. and $V(x) \in L_{\text {loc }}^{\infty}(\mathbb{G})$; one says $V(x) \in$ $B_{\infty}$ if there exists a positive constant $c$ such that

$$
\sup _{B} V(x) \leq c\left(|B|^{-1} \int_{B} V(x) d x\right),
$$

for any metric ball $B$ in $\mathbb{G}$.

It is easy to see that $B_{\infty} \subset B_{q} \subset B_{p}, 1<p<q<\infty$.

\section{Proofs of Theorems 3 and 4}

We first prove Theorem 4 and then prove Theorem 3.

3.1. Proof of Theorem 4. The following lemma is due to Lu and Wheeden $[30,31]$. It will play a key role in our proof.

Lemma 11. Let $B=B_{r_{B}}\left(x_{0}\right)$ be a metric ball in $\mathbb{G}$. If $u \in$ $C^{\infty}(B)$, then there exists a first order polynomial $P_{B}(x)$ such that, for a.e. $x \in B$,

$$
\left|u(x)-P_{B}(x)\right| \leq c \int_{B}\left|X^{2} u(y)\right| \frac{d(x, y)^{2}}{|B(x, d(x, y))|} d y,
$$

where the positive constant $c$ is independent of $u, x$, and $B$. Moreover, if $u \in C_{0}^{\infty}(B)$, then for a.e. $x \in B$,

$$
|u(x)| \leq c \int_{B}\left|X^{2} u(y)\right| \frac{d(x, y)^{2}}{|B(x, d(x, y))|} d y,
$$

where $c>0$ is independent of $u, x$, and $B$.

Proof of Theorem 4. By (31), Fubini's Theorem, and Hölder's inequality, we have

$$
\begin{aligned}
& \int_{B}\left|u(x)-P_{B}(x)\right|^{p}|V(x)| d x \\
& \leq c \int_{B}|V(x)|\left|u(x)-P_{B}(x)\right|^{p-1} \\
& \times\left(\int_{B}\left|X^{2} u(y)\right| \frac{d(x, y)^{2}}{|B(x, d(x, y))|} d y\right) d x \\
& =c \int_{B}\left|X^{2} u(y)\right| \\
& \times\left(\int_{B}|V(x)|\left|u(x)-P_{B}(x)\right|^{p-1}\right. \\
& \left.\times \frac{d(x, y)^{2}}{|B(x, d(x, y))|} d x\right) d y \\
& \leq c\left(\int_{B}\left|X^{2} u(y)\right|^{p} d y\right)^{1 / p} \\
& \cdot\left(\int _ { B } \left(\int_{B}|V(x)|\left|u(x)-P_{B}(x)\right|^{p-1}\right.\right. \\
& \left.\left.\times \frac{d(x, y)^{2}}{|B(x, d(x, y))|} d x\right)^{p /(p-1)} d y\right)^{(p-1) / p} \\
& \equiv c\left(\int_{B}\left|X^{2} u(y)\right|^{p} d y\right)^{1 / p} \cdot I \text {. }
\end{aligned}
$$


Now a computation yields

$$
\begin{aligned}
& I^{p /(p-1)} \\
& \leq \int_{B}\left(\int_{B}|V(z)| \frac{d(z, y)^{2}}{|B(z, d(z, y))|} d z\right)^{1 /(p-1)} \\
& \cdot\left(\int_{B}\left|u(x)-P_{B}(x)\right|^{p}|V(x)|\right. \\
& \left.\times \frac{d(x, y)^{2}}{|B(x, d(x, y))|} d x\right) d y \\
& =\int_{B}\left|u(x)-P_{B}(x)\right|^{p}|V(x)| \int_{B} \frac{d(x, y)^{2}}{|B(x, d(x, y))|} \\
& \cdot\left(\int_{B}|V(z)| \frac{d(z, y)^{2}}{|B(z, d(z, y))|} d z\right)^{1 /(p-1)} d y d x \\
& \leq \int_{B\left(x_{0}, r_{B}\right)}\left|u(x)-P_{B}(x)\right|^{p}|V(x)| \\
& \times \int_{B\left(x, 2 r_{B}\right)} \frac{d(x, y)^{2}}{|B(x, d(x, y))|} \\
& \cdot\left(\int_{B\left(x, 2 r_{B}\right)}|V(z)|\right. \\
& \left.\times \frac{d(z, y)^{2}}{|B(z, d(z, y))|} d z\right)^{1 /(p-1)} d y d x \\
& \leq\left(\varphi_{V}\left(2 r_{B}\right)\right)^{1 /(p-1)} \int_{B}\left|u(x)-P_{B}(x)\right|^{p}|V(x)| d x .
\end{aligned}
$$

Therefore,

$$
\begin{aligned}
\int_{B}\left|u(x)-P_{B}(x)\right|^{p}|V(x)| d x \\
\leq c\left(\varphi_{V}\left(2 r_{B}\right)\right)^{1 / p}\left(\int_{B}\left|u(x)-P_{B}(x)\right|^{p}|V(x)| d x\right)^{(p-1) / p} \\
\quad \times\left(\int_{B}\left|X^{2} u(y)\right|^{p} d y\right)^{1 / p} .
\end{aligned}
$$

It implies (12).

By using (32) and repeating the argument above for (12), we immediately obtain (13).

3.2. Proof of Theorem 3. Let us recall $L^{p}$ estimates for the operator $A=\sum_{i, j=1}^{m} a_{i j} X_{i} X_{j}$ by Bramanti and Brandolini [12, Theorem 2].

Lemma 12. Under the assumptions (4) and (5), for every $p \in(1, \infty)$, there exist positive constants $c=c(p, \mu, \mathbb{G})$ and $r=r(p, \mu, \eta, \mathbb{G})$, where $\eta$ denotes the VMO moduli of coefficients $a_{i j}$, such that, for any $u \in C_{0}^{\infty}(\mathbb{G})$ and sprt $u \subset B_{r}\left(B_{r}\right.$ any metric ball of radius $r$ ),

$$
\left\|X^{2} u\right\|_{L^{p}\left(B_{r}\right)} \leq c\|A u\|_{L^{p}\left(B_{r}\right)} .
$$

Based on it and Theorem 4, we have the following $L^{p}$ estimates for $L$ in (3).

Lemma 13. Under the assumptions (4) and (5), for every $p \in$ $(1, \infty)$ and $V^{p} \in S_{p}(\mathbb{G})$, there exist positive constants $r_{0}=$ $r_{0}(p, \mu, \eta, \mathbb{G})$ and $c=c\left(p, r_{0}, \mu, V, \mathbb{G}\right)$ such that, for any $u \in$ $C_{0}^{\infty}(\mathbb{G})$ and sprt $u \subset B_{r_{0}}$,

$$
\left\|X^{2} u\right\|_{L^{p}\left(B_{r_{0}}\right)}+\|V u\|_{L^{p}\left(B_{r_{0}}\right)} \leq c\|L u\|_{L^{p}\left(B_{r_{0}}\right)} .
$$

Proof. By Theorem 4,

$$
\|V u\|_{L^{p}\left(B_{r_{0}}\right)}^{p} \leq c \varphi_{V^{p}}\left(2 r_{0}\right) \int_{B_{r_{0}}}\left|X^{2} u(x)\right|^{p} d x .
$$

Applying Lemma 12, it follows that

$$
\begin{aligned}
& \left\|X^{2} u\right\|_{L^{p}\left(B_{r_{0}}\right)}+\|V u\|_{L^{p}\left(B_{r_{0}}\right)} \\
& \quad \leq c\left(\|A u\|_{L^{p}\left(B_{r_{0}}\right)}+\|V u\|_{L^{p}\left(B_{r_{0}}\right)}\right) \\
& \quad \leq c\left(\|L u\|_{L^{p}\left(B_{r_{0}}\right)}+\|V u\|_{L^{p}\left(B_{r_{0}}\right)}\right) \\
& \quad \leq c\left(\|L u\|_{L^{p}\left(B_{r_{0}}\right)}+\varphi_{V^{p}}\left(2 r_{0}\right)^{1 / p}\left\|X^{2} u\right\|_{L^{p}\left(B_{r_{0}}\right)}\right) .
\end{aligned}
$$

Choosing $r_{0}>0$ such that $c \varphi_{V^{p}}\left(2 r_{0}\right)^{1 / p} \leq 1 / 2$, we derive (37).

Proof of Theorem 3. We consult the way in [1, pages 342-343] and apply our previous results. By the basic theorem on the partition of unity (e.g., see [32, page 66]), there exists a partition of unity of nonnegative functions $\left\{\varphi_{i}\right\}_{i=1}^{\infty}$ in $\mathbb{G}$ such that $\varphi_{i} \in C_{0}^{\infty}\left(B_{r_{0}}\left(z_{i}\right)\right)$ with $r_{0}$ in Lemma 13 and a family of metric balls $\left\{B_{r_{0}}\left(z_{i}\right)\right\}_{i=1}^{\infty}$ satisfying the finite overlapping property. We have from Lemma 13 that

$$
\begin{aligned}
& \left\|X^{2} u\right\|_{L^{p}(\mathbb{G})}+\|V u\|_{L^{p}(\mathbb{G})} \\
& =\left\|\sum_{i} X^{2}\left(\varphi_{i} u\right)\right\|_{L^{p}(\mathbb{G})}+\left\|\sum_{i} V \varphi_{i} u\right\|_{L^{p}(\mathbb{G})} \\
& \quad \leq c\left(\sum_{i}\left\|X^{2}\left(\varphi_{i} u\right)\right\|_{L^{p}\left(B_{r_{0}}\left(z_{i}\right)\right)}+\sum_{i}\left\|V \varphi_{i} u\right\|_{L^{p}\left(B_{r_{0}}\left(z_{i}\right)\right)}\right) \\
& \quad \leq c \sum_{i}\left\|L\left(\varphi_{i} u\right)\right\|_{L^{p}\left(B_{r_{0}}\left(z_{i}\right)\right)} \\
& \leq \sum_{i}\left(\|L u\|_{L^{p}\left(B_{r_{0}}\left(z_{i}\right)\right)}+\|D u\|_{L^{p}\left(B_{r_{0}}\left(z_{i}\right)\right)}+\|u\|_{L^{p}\left(B_{r_{0}}\left(z_{i}\right)\right)}\right) \\
& \quad \leq c\left(\|L u\|_{L^{p}(\mathbb{G})}+\|X u\|_{L^{p}(\mathbb{G})}+\|u\|_{L^{p}(\mathbb{G})}\right) .
\end{aligned}
$$


Combining with the interpolation inequality (see [12, Proposition 2])

$$
\|X u\|_{L^{p}(\mathbb{G})} \leq \varepsilon\left\|X^{2} u\right\|_{L^{p}(\mathbb{G})}+\frac{2}{\varepsilon}\|u\|_{L^{p}(\mathbb{G})},
$$

$$
\text { for any } \varepsilon>0 \text {, }
$$

we obtain (11).

\section{Proof of Theorem 6}

Several preliminary conclusions are necessary.

Lemma 14. If $V \in B_{q}, q>1$, then there exists a constant $c>0$ such that, for any $1 \leq p<q<\infty$ and $0<r<R<\infty$,

$$
\begin{aligned}
& \frac{1}{\left|B_{r}(x)\right|} \int_{B_{r}(x)} V(y)^{p} d y \\
& \quad \leq c\left(\frac{R}{r}\right)^{p Q / q}\left(\frac{1}{\left|B_{R}(x)\right|} \int_{B_{R}(x)} V(y) d y\right)^{p} .
\end{aligned}
$$

Proof. By Hölder's inequality and (29), it yields

$$
\begin{aligned}
& \frac{1}{\left|B_{r}(x)\right|} \int_{B_{r}(x)} V(y)^{p} d y \\
& \quad \leq\left(\frac{1}{\left|B_{r}(x)\right|} \int_{B_{r}(x)} V(y)^{q} d y\right)^{p / q} \\
& \quad \leq c\left(\frac{R}{r}\right)^{Q}\left(\frac{1}{\left|B_{R}(x)\right|} \int_{B_{R}(x)} V(y)^{q} d y\right)^{p / q} \\
& \quad \leq c\left(\frac{R}{r}\right)^{p Q / q}\left(\frac{1}{\left|B_{R}(x)\right|} \int_{B_{R}(x)} V(y) d y\right)^{p} .
\end{aligned}
$$

Remark 15. If we take $\mathbb{G}=\mathbb{R}^{n}$ and $p=1$, then Lemma 14 gives the version in [2, Lemma 1.2].

Lemma 16. If $V \in B_{q} \cap L^{1}(\mathbb{G}), q>Q / 2$, then $V^{p} \in S_{p}(\mathbb{G})$, $1<p \leq 2 q / Q$.

Proof. For any $x \in \mathbb{G}$, it follows that

$$
\begin{aligned}
& I=: \int_{d(x, y)<r} \frac{d^{2}(x, y)}{|B(x, d(x, y))|} \\
& \quad \times\left(\int_{d(z, x)<r} V^{p}(z) \frac{d^{2}(z, y)}{|B(z, d(z, y))|} d z\right)^{1 /(p-1)} d y \\
& \quad \leq \int_{d(x, y)<r} \frac{d^{2}(x, y)}{|B(x, d(x, y))|} d y \\
& \quad \times\left(\int_{d(z, y)<2 r} V^{p}(z) \frac{d^{2}(z, y)}{|B(z, d(z, y))|} d z\right)^{1 /(p-1)} d y \\
&
\end{aligned}
$$

By (42), it yields

$$
\begin{aligned}
I_{1} & \leq \sum_{k=0}^{\infty} \int_{r / 2^{k} \leq d(z, y)<r / 2^{k-1}} V^{p}(z) \frac{d^{2}(z, y)}{|B(z, d(z, y))|} d z \\
& \leq c \sum_{k=0}^{\infty}\left(\frac{r}{2^{k}}\right)^{2-\mathrm{Q}} \int_{d(z, y)<r / 2^{k-1}} V^{p}(z) d z \\
& \leq c r^{2-p \mathrm{Q} / q} R^{p \mathrm{Q} / q-p \mathrm{Q}} \sum_{k=0}^{\infty}\left(\frac{1}{2^{k}}\right)^{2-p \mathrm{Q} / q}\left(\int_{B(y, R)} V(z) d z\right)^{p} \\
& \leq c r^{2-p \mathrm{Q} / q} R^{p \mathrm{Q} / q-p \mathrm{Q}}\left(\int_{\mathbb{G}} V d z\right)^{p} .
\end{aligned}
$$

Also, we have

$$
\begin{aligned}
& \int_{d(x, y)<r} \frac{d^{2}(x, y)}{|B(x, d(x, y))|} d y \\
& \quad \leq \sum_{k=1}^{\infty} \int_{r / 2^{k} \leq d(x, y)<r / 2^{k-1}} \frac{d^{2}(x, y)}{|B(x, d(x, y))|} d y \\
& \quad \leq c \sum_{k=1}^{\infty}\left(\frac{r}{2^{k}}\right)^{2-Q} \int_{d(x, y)<r / 2^{k-1}} d y \\
& \quad \leq c r^{2} .
\end{aligned}
$$

Therefore combining (45) and (46) gets

$$
\begin{aligned}
I \leq & c r^{(1 /(p-1))(2-p \mathrm{Q} / q)} R^{(p \mathrm{Q} /(p-1))(1 / q-1)} \\
& \times\left(\int_{\mathbb{G}} V(z) d z\right)^{p /(p-1)} \cdot c r^{2} \\
\leq & c r^{(1 /(p-1))(2 p-p \mathrm{Q} / q)} R^{(p \mathrm{Q} /(p-1))(1 / q-1)} \\
& \times\left(\int_{\mathbb{G}} V(z) d z\right)^{p /(p-1)} \longrightarrow 0 \\
\text { as } r & \longrightarrow 0 .
\end{aligned}
$$

The result is proved.

Proof of Theorem 6. By Lemma 16 and Theorem 3, we immediately obtain Theorem 6.

Remark 17. In order to assure the convergence of the series $\sum_{k=0}^{\infty}\left(2^{-k}\right)^{2-p Q / q}$ in the proof of Lemma 16 , we require the assumption $p \leq 2 q / Q$, which leads to the range of $p$ in Theorem 6 smaller than [1, Theorem 1].

\section{Results to the Euclidean Case and Elliptic Operators}

Here for convenience of readers, we restate Theorems 3 and 4 corresponding to the Euclidean case but omit their proofs because the proofs are analogous to Theorems 3 and 4 . It will be assumed for the leading coefficients $a_{i j}$ in (1) that 
$\left(H_{1}\right) a_{i j}(x)=a_{j i}(x) \in L^{\infty}\left(\mathbb{R}^{n}\right)$ for all $i, j=1, \ldots, n$ and there exists a positive constant $\mu$ such that, for any $x \in \mathbb{R}^{n}$ and $\xi \in \mathbb{R}^{n}$,

$$
\mu^{-1}|\xi|^{2} \leq \sum_{i, j=1}^{n} a_{i j}(x) \xi_{i} \xi_{j} \leq \mu|\xi|^{2}
$$

$\left(H_{2}\right) a_{i j}(x) \in \operatorname{VMO}\left(\mathbb{R}^{n}\right)$; that is, for $i, j=1, \ldots, n$,

$$
\begin{array}{r}
\eta_{i j}=\sup _{\rho \leq r} \sup _{x \in \mathbb{R}^{n}}\left(\left|B_{\rho}(x)\right|^{-1} \int_{B_{\rho}(x)}\left|a_{i j}(y)-a_{i j}^{B}\right| d y\right) \\
\text { as } r \longrightarrow 0,
\end{array}
$$

where $a_{i j}^{B}=\left|B_{\rho}(x)\right|^{-1} \int_{B_{\rho}(x)} a_{i j}(y) d y$.

A function $V \in S_{p}\left(\mathbb{R}^{n}\right)$ for $1<p<\infty$ means that, for each $r>0$,

$$
\begin{aligned}
\varphi_{V}(r):=\sup _{x \in \mathbb{R}^{n}}\left(\int_{|x-y|<r} \frac{1}{|x-y|^{n-2}}\right. \\
\left.\quad \times\left(\int_{|z-y|<r} \frac{|V(z)|}{|z-y|^{n-2}} d z\right)^{1 /(p-1)} d y\right)^{p-1}
\end{aligned}
$$

is finite and

$$
\lim _{r \rightarrow 0} \varphi_{V}(r)=0
$$

Theorem 18. Under assumptions $\left(H_{1}\right)$ and $\left(H_{2}\right)$, if $V^{p} \in$ $S_{p}\left(\mathbb{R}^{n}\right), 1<p<\infty$, then there exists a positive constant $c=c(n, p, \mu, \eta, V)$ such that, for any $u \in C_{0}^{\infty}\left(\mathbb{R}^{n}\right)$, one has

$$
\begin{aligned}
& \|u\|_{W^{2, p}\left(\mathbb{R}^{n}\right)}+\|V u\|_{L^{p}\left(\mathbb{R}^{n}\right)} \\
& \quad \leq c\left(\|\boldsymbol{Q} u\|_{L^{p}\left(\mathbb{R}^{n}\right)}+\|u\|_{L^{p}\left(\mathbb{R}^{n}\right)}\right),
\end{aligned}
$$

where $\eta$ depends only on the VMO moduli of the coefficients $a_{i j}$.

Remark 19. If $|V| \equiv 0, x \in \mathbb{R}^{n}$ or $|V| \leq$ const., $x \in \mathbb{R}^{n}$, the $L^{p}$ theory of (1) with discontinuous leading coefficients was intensively studied and the result was proved in [33-36] and so forth. Bramanti et al. [1] obtained a prior $L^{p}\left(\mathbb{R}^{n}\right)$ estimate for (1) with $a_{i j} \in V M O$ and $V \in B_{q}$. Here $B_{q} \not \subset S_{p}\left(\mathbb{R}^{n}\right)$ and $S_{p}\left(\mathbb{R}^{n}\right) \not \subset B_{q}$.

Theorem 20. Let $B=B_{r_{B}}\left(x_{0}\right)$ be any ball in $\mathbb{R}^{n}$. If $V \in$ $S_{p}\left(\mathbb{R}^{n}\right)$, then there exists a first order polynomial $P_{B}(x)$ in $\mathbb{R}^{n}$ such that, for any $u \in C^{\infty}(B)$, one has

$$
\int_{B}\left|u-P_{B}(x)\right|^{p}|V| d x \leq c \varphi_{V}\left(2 r_{B}\right) \int_{B}\left|X^{2} u\right|^{p} d x,
$$

where the positive constant $c$ is independent of $u$ and $B$. Moreover, for any $u \in C_{0}^{\infty}(B)$, one has

$$
\int_{B}|u|^{p}|V| d x \leq c \varphi_{V}\left(2 r_{B}\right) \int_{B}\left|X^{2} u\right|^{p} d x
$$

where $c>0$ is independent of $u$ and $B$.

\section{Conflict of Interests}

The authors declare that there is no conflict of interests regarding the publication of this paper.

\section{Acknowledgments}

The first author was supported by the National Natural Science Foundation of China (Grant no. 11271299) and Natural Science Foundation Research Project of Shaanxi Province (Grant no. 2012JM1014). The authors are very grateful to the anonymous referee who read carefully the manuscript and offered valuable suggestions.

\section{References}

[1] M. Bramanti, L. Brandolini, E. Harboure, and B. Viviani, "Global $W^{2, p}$ estimates for nondivergence elliptic operators with potentials satisfying a reverse Hölder condition," Annali di Matematica Pura ed Applicata, vol. 191, no. 2, pp. 339-362, 2012.

[2] Z. W. Shen, " $L^{p}$ estimates for Schrödinger operators with certain potentials," Annales de L'Institut Fourier, vol. 45, no. 2, pp. 513546, 1995.

[3] S. Thangavelu, "Riesz transforms and the wave equation for the Hermite operator," Communications in Partial Differential Equations, vol. 15, no. 8, pp. 1199-1215, 1990.

[4] C. Vitanza, "A new contribution to the $W^{2, p}$-regularity for a class of elliptic second order equations with discontinuous coefficients," Le Matematiche, vol. 47, no. 1, pp. 177-186, 1992.

[5] J. Zhong, Harmonic analysis for some Schrödinger type operators [Ph.D. thesis], Princeton University, Princeton, NJ, USA, 1993.

[6] G. Di Fazio and P. Zamboni, "A Fefferman-Poincaré type inequality for Carnot-Carathéodory vector fields," Proceedings of the American Mathematical Society, vol. 130, no. 9, pp. 26552660, 2002

[7] M. A. Ragusa and P. Zamboni, "A potential theoretic inequality," Czechoslovak Mathematical Journal, vol. 51(126), no. 1, pp. 5565, 2001.

[8] G. Citti, N. Garofalo, and E. Lanconelli, "Harnack's inequality for sum of squares of vector fields plus a potential," The American Journal of Mathematics, vol. 115, no. 3, pp. 699-734, 1993.

[9] H.-Q. Li, "Estimations $L^{p}$ des opérateurs de Schrödinger sur les groupes nilpotents," Journal of Functional Analysis, vol. 161, no. 1, pp. 152-218, 1999.

[10] G. Lu, "A Fefferman-Phong type inequality for degenerate vector fields and applications," Panamerican Mathematical Journal, vol. 6, no. 4, pp. 37-57, 1996.

[11] G. Lu, "On Harnack's inequality for a class of strongly degenerate Schrödinger operators formed by vector fields," Differential and Integral Equations, vol. 7, no. 1, pp. 73-100, 1994. 
[12] M. Bramanti and L. Brandolini, " $L^{p}$ estimates for uniformly hypoelliptic operators with discontinuous coefficients on homogeneous groups," Università e Politecnico di Torino. Seminario Matematico. Rendiconti, vol. 58, no. 4, pp. 389-433 (2003), 2000.

[13] X. Feng and P. Niu, "Global Orlicz regularity for sub-Laplace equations on homogeneous groups," Bulletin des Sciences Mathematiques, vol. 136, no. 6, pp. 648-665, 2012.

[14] X. Feng and P. Niu, "Interior regularity for degenerate elliptic equations with drift on homogeneous groups," Journal of Lie Theory, vol. 23, no. 3, pp. 803-825, 2013.

[15] C. L. Fefferman, "The uncertainty principle," Bulletin of the American Mathematical Society, vol. 9, no. 2, pp. 129-206, 1983.

[16] F. Chiarenza and M. Frasca, "A remark on a paper by C. Fefferman," Proceedings of the American Mathematical Society, vol. 108, no. 2, pp. 407-409, 1990.

[17] D. Danielli, "A Fefferman-Phong type inequality and applications to quasilinear subelliptic equations," Potential Analysis, vol. 11, no. 4, pp. 387-413, 1999.

[18] D. Danielli, N. Garofalo, and D.-M. Nhieu, "Trace inequalities for Carnot-Carathéodory spaces and applications," Annali della Scuola Normale Superiore di Pisa, vol. 27, no. 2, pp. 195-252, 1998.

[19] G. Lu, "Embedding theorems into Lipschitz and BMO spaces and applications to quasilinear subelliptic differential equations," Publicacions Matemàtiques, vol. 40, no. 2, pp. 301-329, 1996.

[20] M. Bramanti and L. Brandolini, " $L^{P}$ estimates for nonvariational hypoelliptic operators with VMO coefficients," Transactions of the American Mathematical Society, vol. 352, no. 2, pp. 781-822, 2000.

[21] A. Calderón, "Inequalities for the maximal function relative to a metric," Studia Mathematica, vol. 57, no. 3, pp. 297-306, 1976.

[22] A. Bonfiglioli, E. Lanconelli, and F. Uguzzoni, Stratified Lie Groups and Potential Theory for Their Sub-Laplacians, Springer Monographs in Mathematics, Springer, Berlin, Germany, 2007.

[23] G. B. Folland, "Subelliptic estimates and function spaces on nilpotent Lie groups," Arkiv för Matematik, vol. 13, no. 2, pp. 161207, 1975.

[24] G. B. Folland and E. M. Stein, Hardy Spaces on Homogeneous Groups, Princeton University Press, Princeton, NJ, USA, 1980.

[25] E. M. Stein, Harmonic Analysis: Real-Variable Methods, Orthogonality and Oscillatory Integrals, Princeton University Press, Princeton, NJ, USA, 1993.

[26] L. Hörmander, "Hypoelliptic second order differential equations," Acta Mathematica, vol. 119, pp. 147-171, 1967.

[27] W. S. Cohn, G. Lu, and P. Wang, "Sub-elliptic global high order Poincaré inequalities in stratified Lie groups and applications," Journal of Functional Analysis, vol. 249, no. 2, pp. 393-424, 2007.

[28] A. Nagel, E. M. Stein, and S. Wainger, "Balls and metrics defined by vector fields. I: basic properties," Acta Mathematica, vol. 155, no. 1, pp. 130-147, 1985.

[29] W.-L. Chow, "Über Systeme von liearren partiellen Differentialgleichungen erster Ordnung," Mathematische Annalen, vol. 117, pp. 98-105, 1939.

[30] G. Lu and R. L. Wheeden, "High order representation formulas and embedding theorems on stratified groups and generalizations," Studia Mathematica, vol. 142, no. 2, pp. 101-133, 2000.

[31] G. Lu and R. L. Wheeden, "Simultaneous representation and approximation formulas and high-order Sobolev embedding theorems on stratified groups," Constructive Approximation, vol. 20, no. 4, pp. 647-668, 2004.

[32] W. Chen, An Introduction to Differentiable Manifold, Higher Education Press, Beijing, China, 2nd edition, 2001.

[33] F. Chiarenza, M. Frasca, and P. Longo, "Interior $W^{2, p}$ estimates for nondivergence elliptic equations with discontinuous coefficients," Ricerche di Matematica, vol. 40, no. 1, pp. 149-168, 1991.

[34] F. Chiarenza, M. Frasca, and P. Longo, " $W^{2, p}$-solvability of the Dirichlet problem for nondivergence elliptic equations with VMO coefficients," Transactions of the American Mathematical Society, vol. 336, no. 2, pp. 841-853, 1993.

[35] D. Kim and N. V. Krylov, "Elliptic differential equations with coefficients measurable with respect to one variable and VMO with respect to the others," SIAM Journal on Mathematical Analysis, vol. 39, no. 2, pp. 489-506, 2007.

[36] N. V. Krylov, "Second-order elliptic equations with variably partially VMOcoefficients," Journal of Functional Analysis, vol. 257, no. 6, pp. 1695-1712, 2009. 


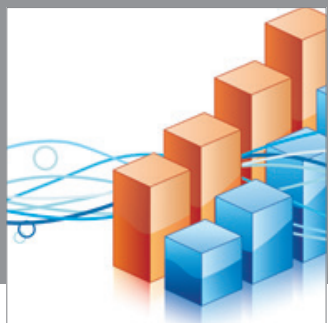

Advances in

Operations Research

mansans

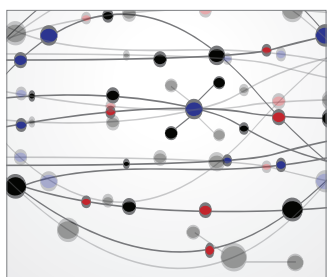

The Scientific World Journal
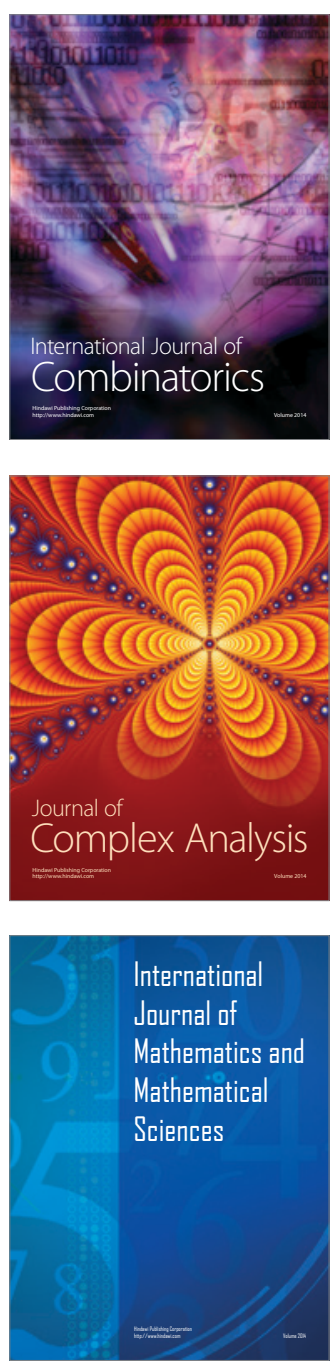
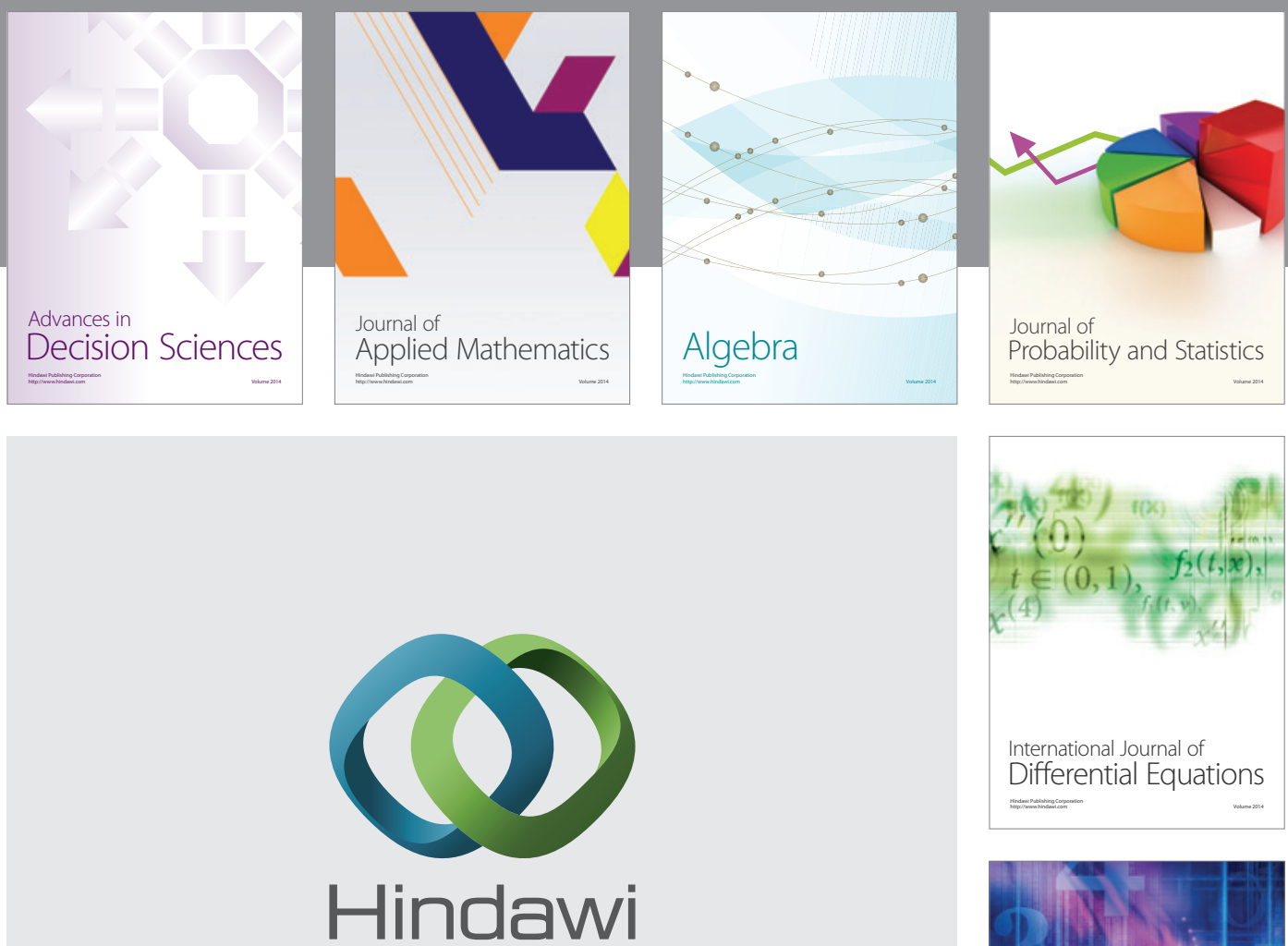

Submit your manuscripts at http://www.hindawi.com
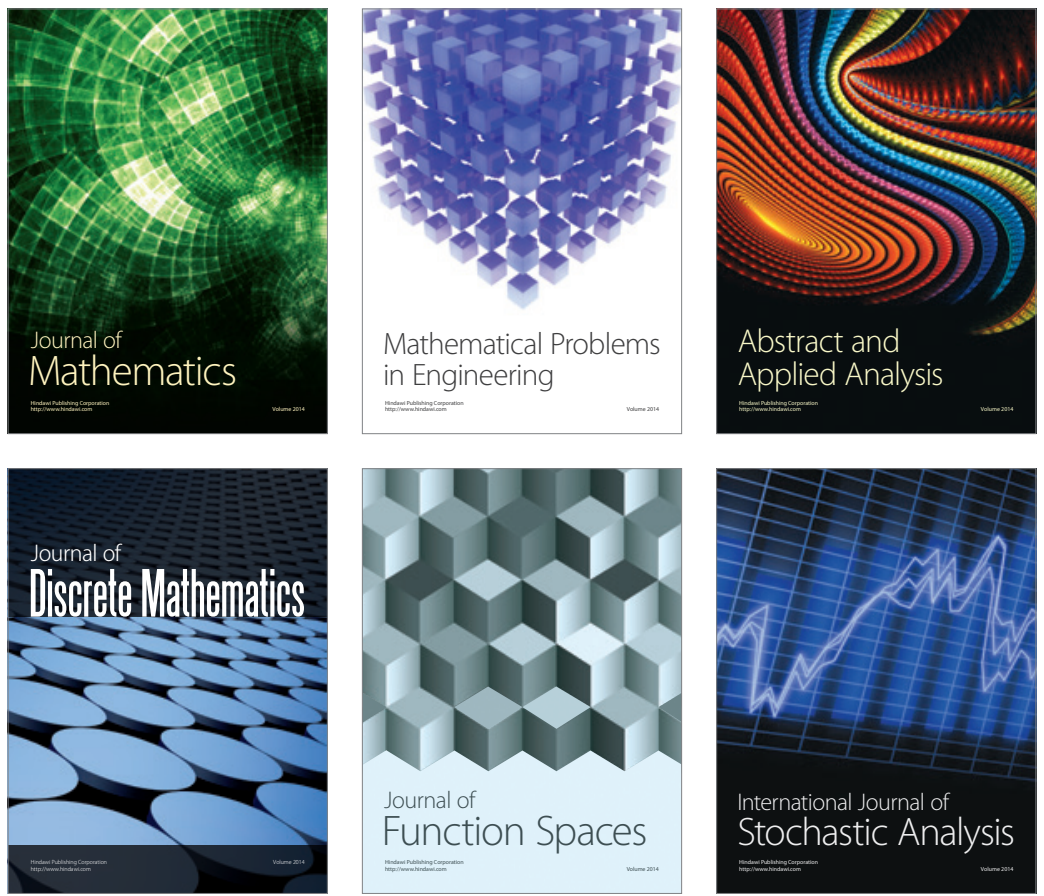

Journal of

Function Spaces

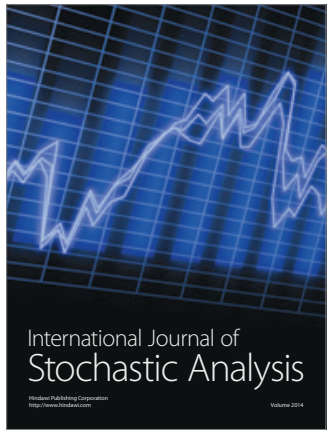

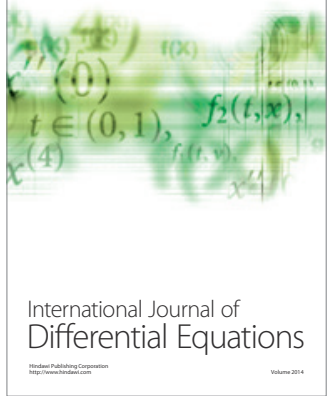
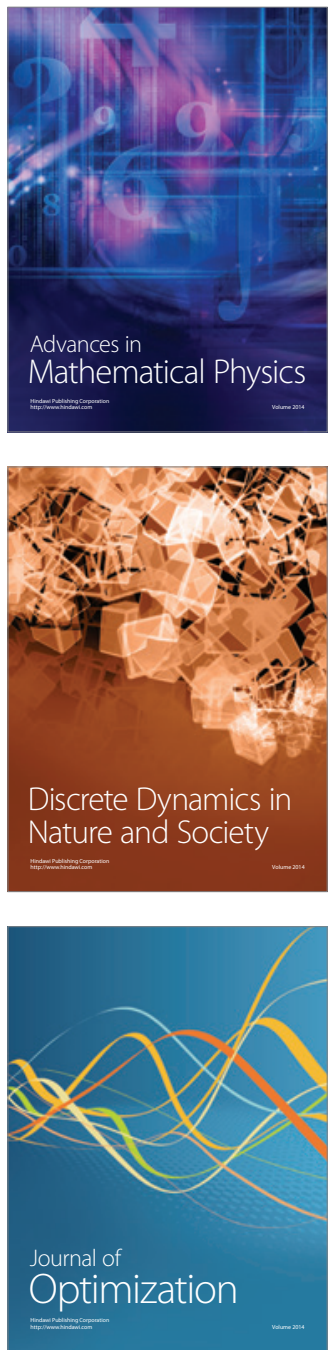